\title{
Concordancia de tres escalas de riesgo cardiovascular en mujeres con diabetes mellitus tipo 2
}

Concordance of three scales of cardiovascular risk in women with diabetes mellitus type 2

\author{
${ }^{\oplus}$ Elkin Navarro Quiroz ${ }^{1},{ }^{\oplus}$ Engelbert Peña Merlano ${ }^{1},{ }^{\oplus}$ Anoris Fernández Gutiérrez ${ }^{2}$, ${ }^{\circ}$ Roberto Navarro Quiroz ${ }^{3}$, \\ ${ }^{\circ}$ Emilio Abuabara ${ }^{4}{ }^{\oplus}$ Jeison Enrique Torrens Soto ${ }^{4}{ }^{\bullet}$ Germán Arenas ${ }^{5},{ }^{\oplus J}$ Juan Pablo Zakzuk Pérez ${ }^{5}$,

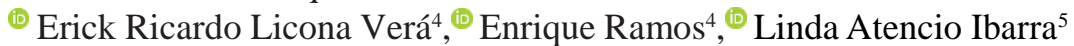 \\ 'Facultad de Ciencias Básicas y Biomédicas, Universidad Simón Bolívar, Barranquilla, Colombia. \\ ${ }^{2}$ Facultad de Medicina, Universidad Cooperativa de Colombia, Sede Santa Marta, Santa Marta, Colombia. \\ ${ }^{3}$ Universidade federal do ABC, São Pablo, Brasil. \\ ${ }^{4}$ Grupo de investigación GIBACUS, Universidad del Sinú, Seccional Cartagena, Cartagena, Colombia. \\ ${ }^{5}$ Facultad de Medicina, Universidad de Cartagena, Cartagena, Colombia.
}

\begin{abstract}
Resumen
Introducción: establecer estimadores del riesgo cardiovasculares (RCV) que permitan tener igual concordancia dentro del subconjunto de la población es importante para diseñar terapias que briden ventajas a cada subpoblación.

Objetivo: comparar las escalas Framingham, SCORE/REGICOR y ACC/AHA en una corte de mujeres con diabetes.

Materiales y métodos: se realizó un estudio descriptivo transversal en donde se compararon las escalas Framingham, SCORE/ REGICOR y ACC/AHA para identificar los niveles de riesgo cardiovascular en mujeres con diabetes mellitus tipo 2. Los datos se recopilaron entre 2014 y 2016 en pacientes del Centro Endocrinológico del Caribe ubicado en Barranquilla, Colombia. Se consideraron variables sociodemográficas, antropométricas y clínicas.

Resultados: el 100\% de la muestra (n=107) eran mujeres con una edad media de 58,5 $\pm 13,646$ años. Según la escala Framingham el $72,9 \%(n=78)$ se clasificó como de riesgo bajo; el 21,5\% (23), intermedio, y el 5,6\% ( $n=6)$, alto. Con la escala SCORE/REGICOR el $57,0 \%(n=61)$ estuvo en riesgo bajo; el 42,1\% ( $n=45)$, en moderado, y el 0,9\% $(n=1)$, en alto. Finalmente, mediante la escala ACC/ AHA el 35,5\% (n=38) se clasificó en riesgo bajo y el 64,5\% ( $n=69)$, en elevado.

Conclusiones: en el presente estudio se observó una baja concordancia al comparar las escalas de riesgo cardíaco analizadas (Framingham, SCORE / REGICOR y ACC / AHA) en mujeres colombianas con diabetes mellitus tipo dos y se observó además que la edad y el colesterol total fueron los valores que más afectaron la variación entre las escalas comparadas.

Palabras clave: enfermedades cardiovasculares, diabetes mellitus tipo 2, factores de riesgo (DeCS).
\end{abstract}

https://doi.org/10.22265/acnef.7.2.429

\begin{abstract}
Introduction: Establishing cardiovascular risk (CVR) estimators that allow for equal concordance within the subset of the population is important for establishing therapy that provide advantages for each subpopulation.In this article we focus on buying CVR scale in women with type 2 diabetes mellitus .

Objective: Compare between the Framingham, SCORE / REGICOR and ACC / AHA scales in a court of women with diabetes. Methods: The data collected were between 2014 and 2016 from the population of the Caribbean Endocrinological Center, Barranquilla, Colombia, considering the sociodemographic, anthropometric, and clinical variables. In this study, it was possible to establish comparisons between the Framingham, SCORE/REGICOR and ACC / AHA scales to identify cardiovascular risk levels in women with T2DM.

Results: $100 \%$ of the sample $(n=107)$ were women with a mean age of $58.5 \pm 13,646$ years, $78(72.9 \%)$ were classified as low risk, $23(21.5 \%)$ intermediate and 6 (5.6\%) high with the scale The SCORE/REGICOR scale showed $61(57.0 \%)$ at low risk, $45(42.1 \%)$ moderate and $1(0.9 \%)$ high; while ACC / AHA 38 (35.5\%) is classified as low risk and 69 (64.5\%) as high.

Conclusions: In the present study, a low concordance was observed when comparing the cardiac risk scales (Framingham, SCORE / REGICOR and ACC / AHA) in Colombian women with type two diabetes and it was also observed that age and total cholesterol were the values that more affected variation between the comparative cardiovascular risk scale.
\end{abstract}

Keywords: Cardiovascular Diseases, type 2 diabetes mellitus, risk factors (MeSH).

https://doi.org/10.22265/acnef.7.2.429

Citación: Concordancia de tres escalas de riesgo cardiovascular en mujeres con diabetes mellitus tipo 2. Navarro Quiroz E, Peña Merlano E, Fernández Gutiérrez A, Navarro Quiroz A, Abuabara E, Torrens Soto JE, et al. Rev. Colomb. Nefrol. 2020;7(2):30-36. https://doi.org/10.22265/acnef.7.2.429

Recibido: 15.04.20, Aceptado: 22.05.20, Publicado en línea: 22.05.20

Correspondencia: Roberto Carlos Navarro Quiroz, robertcnavarro@gmail.com 


\section{Introducción}

$\mathrm{L}$ a diabetes mellitus tipo 2 (DM2) es una enfermedad con alta prevalencia a nivel mundial cuyos cambios metabólicos se han asociado con alteraciones bioquímicas y de marcadores clínicos, por lo cual causa complicaciones que van desde discapacidad temporal hasta secuelas permanentes; además, dados los efectos que provoca, tiene un alto impacto en la economía de los Estados y en los sistemas de salud; por ejemplo, en países europeos los costos destinados para cuidados de DM2 son elevados en comparación con los de otras enfermedades crónicas ${ }^{1}$.

De acuerdo con lo descrito por la Organización Mundial de la Salud (OMS), citada por Roglic ${ }^{2}$, en 2014 el 8,5\% de los adultos tuvieron diabetes, en 2015 1,6 millones de personas murieron como resultado directo de esta patología y en 2012 la hiperglicemia causo 2,2 millones de muertes.

La DM2 tiene carácter hereditario: los pacientes con uno de los padres diabético tienen el doble de riesgo de presentar diabetes respecto al resto de la población y en aquellos que tienen los dos padres diabéticos el riesgo se eleva seis veces ${ }^{3}$. Estudios recientes intentan explicar parte de esto identificando al menos cuarenta polimorfismos de nucleótidos asociados con un incremento del riesgo de presentar $\mathrm{DM} 2^{4}$.

La DM2 se asocia con un incremento en el riesgo de complicaciones de tipo cardiovascular como arterioesclerosis, infarto agudo de miocardio, accidente cerebrovascular, enfermedad renal y enfermedad arterial periférica ${ }^{5}$, por lo que los pacientes con esta enfermedad tienen el doble riesgo de desarrollar enfermedad coronaria crónica respecto a pacientes no diabéticos. Muchas guías de manejo de DM2 han usado herramientas para valorar el riesgo cardiovascular usando predictores tradicionales como hipertensión, dislipidemia, hemoglobina glicosilada, albuminuria, obesidad, consumo de cigarrillo e historia familiar de enfermedades cardiovasculares ${ }^{6}$, pero es el índice de masa corporal (IMC) el principal factor asociado con DM2 y el que determina un alto riesgo de enfermedad cerebrovascular ${ }^{7}$.
Durante 79 años, el estudio Framinghan se ha usado para calcular el riesgo de enfermedades cardiovasculares y sus factores desencadenantes ${ }^{8}$. Asimismo, en Europa la escala REGICOR ha sido la base de modelos desarrollados, y en Suramérica se incluye la condición socioeconómica ${ }^{9,10}$. Estos estudios han demostrado un mayor riesgo cardiovascular en la población masculina, sin embargo en las mujeres este riesgo ha estado mimetizado por otras enfermedades, principalmente por la patología oncológica, sin tener en cuenta que la principal causa de muerte en mujeres posmenopáusicas es la enfermedad cardiovascular, dentro de la cual la enfermedad coronaria juega un papel protagónico.

El estudio prospectivo de epidemiología urbana y rural (PURE) - Colombia ${ }^{11}$, que se implementó en 11 departamentos del país e incluyó 7.500 adultos de 35 a 70 años, informó una prevalencia de prediabetes del 11,9\%, mientras que el estudio de López-Jaramillo et al. ${ }^{12}$, realizado en adultos de ambos sexos de Barranquilla, Colombia, encontró una prevalencia de intolerancia a la glucosa aislada del 8\%, glucemia en ayunas alterada del $11 \%$ y prevalencia de prediabetes con un primer infarto de miocardio del $29,6 \%$; estos datos demostraron la importancia de la prediabetes tanto por su alta prevalencia entre la población adulta colombiana, como por su relación con los resultados cardiovasculares ${ }^{12}$.

En cuanto a la situación actual de la estimación del riesgo cardiovascular en Colombia, en 2009 Navarrete et al. ${ }^{13}$ realizaron un estudio de prevalencia de factores de riesgo para enfermedad cardiovascular en una muestra de pacientes con hipertensión arterial esencial y demostraron que la coexistencia de hipertensión arterial más diabetes incrementó la prevalencia de patologías renales, hipertrofia ventricular izquierda, enfermedad coronaria y enfermedad vascular periférica. Asimismo, en 2013, Machado-Alba \& Machado-Duque $^{14}$ estimaron el riesgo cardiovascular en pacientes con dislipidemia afiliados al sistema de salud en Colombia, utilizando para esto el algoritmo de Framingham, y encontraron que en mujeres era del 56,4\% $(n=311)$.

Por otra parte, en Colombia no hay estudios en los cual se compare tres escalas de riesgo en una población de pacientes femeninos con DM2, lo cual permitirá tener información valiosa en cuanto al diagnóstico 
y la prevención de riesgo cardiovascular en esta población, por lo cual el presente estudio tiene como objetivo estimar la concordancia de tres clasificaciones de riesgo cardiovascular en mujeres con diagnóstico de DM2 que consultaron a una institución especializada en Barranquilla.

\section{Materiales y métodos}

Se realizó un estudio observacional analítico de concordancia diagnóstica en una población de mujeres con diagnóstico confirmado de DM2 que consultaron al Centro Endocrinológico del Caribe, ubicado en Barranquilla, entre los años 2014 y 2016, y que aceptaron participar en el estudio mediante la firma voluntaria del consentimiento informado. Se excluyeron las pacientes que tuvieran diagnóstico de otra enfermedad metabólica e inmunológica y la muestra final estuvo conformada por 107 mujeres.

Se midieron variables sociodemográficas (edad y estrato socioeconómico), antropométricas (IMC con la cualificación del estado nutricional y antecedente de tabaquismo) y clínicas (cifras de presión arterial, perfil lipídico completo, glicemia y hemoglobina glicosilada (HbA1c)), y se definió el control glucémico con valores de $\mathrm{HbA} 1 \mathrm{c}<7 \%$ para calcular el riesgo cardiovascular por las escalas de Framingham, REGICOR y ACC/AHA.

En la escala de Framingham un puntaje $>10$ fue considerado de riesgo alto; entre 5 y 10, intermedio, y $<5$, bajo. En la escala REGICOR un puntaje $>20$ fue considerado de riesgo alto; entre 10 y 20, intermedio, y $<10$, bajo. En la escala ACC/AHA se clasificó como riesgo bajo a los puntajes $\leq 7,5$ y alto a los puntajes mayores a este punto de corte.

El análisis descriptivo de variables cualitativas se realizó mediante el cálculo de frecuencias absolutas y relativas, mientras que el de las cuantitativas con mediana y rango intercuartílico (RIC). Se estimó el coeficiente de concordancia Kappa de Cohen (k) entre las tres escalas evaluadas para ponderar el riesgo alto y bajo; la concordancia del riesgo intermedio se realizó solo entre la escala de Framingham y REGICOR. En todos los casos se calcularon intervalos de confianza al 95\% (IC95\%) y se definió la fuerza de concordancia pobre con valores de $\mathrm{k}<0,020$; débil entre 0,21 y 0,40 ; moderada entre 0,41 y 0,60 ; buena entre 0,61 y 0,80 y muy buena entre 0,81 y 1,00 .

\section{Resultados}

En el periodo durante el cual se realizó el estudio se identificaron 107 mujeres con DM2; todas aceptaron la participación en el mismo. La mediana de edad fue de 57 años (RIC 48-70) y el estrato de residencia más frecuentemente habitado fue medio con $67,3 \%$ seguido de bajo con $29 \%$. La mediana de IMC de la muestra fue 29,6 (RIC: 26-36,2) y la obesidad fue el estado nutricional más frecuente $(49,5 \%)$, predominando la obesidad grado 2 con $22,4 \%$ seguida de la grado 1 con $16,8 \%$ y la grado 3 con 10,3\%; los otros estados nutricionales en orden de frecuencia fueron sobrepeso $(31,8 \%)$, normopeso $(17,8 \%)$ y delgadez $(0,9 \%)$ (Tabla 1$)$.

La evaluación clínica mostró medianas de tensión sistólica y diastólica al momento de la consulta de 120 y $80 \mathrm{mmHg}$, respectivamente. Por su parte, la mediana de colesterol total fue $198 \mathrm{mg} / \mathrm{dL}$ (RIC: 168-236) y las de colesterol HDL, LDL y VLDL fueron 43, 124 y $30,8 \mathrm{mg} / \mathrm{dL}$, respectivamente; la razón de colesterol total/HDL tuvo una mediana de 456 (RIC: 370-547); los triglicéridos, la glicemia y la hemoglobina glicosilada tuvieron medianas de 154 (RIC: 110-189), $123 \mathrm{mg} / \mathrm{dL}$ (RIC: 104-157) y 7,0\% (RIC 6,2-8,6), respectivamente, lo que evidenció que el 51,4\% ( $\mathrm{n}=55)$ de los pacientes se encontraba en control metabólico (Tabla 2).

La evaluación de riesgo cardiovascular utilizando las tres escalas de riesgo mostró para la escala de Framingham un riesgo alto en 5,6\% de la muestra, moderado en $21,5 \%$ y bajo en $72,9 \%$; la escala REGICOR mostró riesgo alto en $0,9 \%$, intermedio en $42,1 \%$ y bajo en $57 \%$, y finalmente la escala ACC/AHA mostró riesgo alto en $64,5 \%$ y bajo en $35,5 \%$ (Tabla 3 ).

Al estimar la concordancia de riesgo alto en las tres escalas se observaron rangos $<0,2$ en las comparaciones de todas las escalas, lo que evidenció una pobre concordancia (Tabla 4). En específico, al evaluar la concordancia de riesgo bajo comparando la escala Framingham con la REGICOR se obtuvo un 
Tabla 1. Características generales y antecedentes de riesgo.

\begin{tabular}{|c|c|c|c|}
\hline \multirow{2}{*}{\multicolumn{2}{|c|}{ Características }} & \multicolumn{2}{|c|}{ Hallazgos } \\
\hline & & Media & RIC \\
\hline \multicolumn{2}{|c|}{ Edad } & 57 & $(48-70)$ \\
\hline \multirow{2}{*}{\multicolumn{2}{|c|}{ IMC }} & 29,6 & $(26,0-36,2)$ \\
\hline & & $\mathbf{n}$ & $\%$ \\
\hline \multirow{3}{*}{ Estrato } & Alto & 4 & 3,7 \\
\hline & Medio & 72 & 67,3 \\
\hline & Bajo & 31 & 29,0 \\
\hline \multirow{4}{*}{ Estado nutricional } & Delgadez & 1 & 0,9 \\
\hline & Normal & 19 & 17,8 \\
\hline & Sobrepeso & 34 & 31,8 \\
\hline & Obesidad & 53 & 49,5 \\
\hline \multirow{3}{*}{ Grado de obesidad } & 1 & 18 & 16,8 \\
\hline & 2 & 24 & 22,4 \\
\hline & 3 & 11 & 10,3 \\
\hline Tabaquismo & & 7 & 6,5 \\
\hline
\end{tabular}

RIC: rango intercuartílico; IMC: índice de masa corporal. Fuente: elaboración propia.

Tabla 2. Hallazgos paraclínicos de control de las pacientes estudiadas.

\begin{tabular}{|l|c|c|}
\hline \multicolumn{1}{|c|}{ Paraclínico } & Media & RIC \\
\hline TAS Me (RIC) & 120 & $120-130$ \\
\hline TAD Me (RIC) & 80 & $70-80$ \\
\hline Colesterol total (mg/dl) & 198 & $168-236$ \\
\hline HDL & 43,0 & $38,0-51,0$ \\
\hline LDL & 124,0 & $89,2-165,6$ \\
\hline VLDL & 30,8 & $22,0-37,8$ \\
\hline COLTOT/HDL & 4,56 & $3,70-5,47$ \\
\hline TGC & 154 & $110-189$ \\
\hline Glicemia & 123 & $104-167$ \\
\hline HbA1c & 7,0 & $6,2-8,6$ \\
\hline Control HbA1c $\mathrm{n}(\%)$ & $55(51,4)$ & - \\
\hline
\end{tabular}

RIC: rango intercuartílico. Fuente: elaboración propia.

Tabla 3. Valoración del riesgo cardiovascular en las pacientes diabéticas por las tres escalas de riesgo.

\begin{tabular}{|l|l|c|c|c|}
\hline \multirow{2}{*}{ Escalas de riesgo } & \multicolumn{2}{c|}{ Valoración del riesgo cardiovascular n (\%) } \\
\cline { 3 - 5 } & & Alto & Intermedio/moderado & Bajo \\
\hline \multirow{2}{*}{ Framingham } & Rango & $>10$ & $5-10$ & $<5$ \\
\cline { 2 - 5 } & Interpretación & $6(5,6)$ & $23(21,5)$ & $78(72,9)$ \\
\hline \multirow{2}{*}{ REGICOR } & Rango & $>20$ & $10-20$ & $<10$ \\
\cline { 2 - 5 } & Interpretación & $1(0,9)$ & $45(42,1)$ & $\leq 7,57,0)$ \\
\hline \multirow{2}{*}{ ACC/AHA } & Rango & $>7,5$ & \multirow{2}{*}{ NA } & $38(35,5)$ \\
\cline { 2 - 3 } & Interpretación & $69(64,5)$ & &
\end{tabular}

Fuente: elaboración propia. 
Tabla 4. Concordancia de riesgo alto en las tres escalas.

\begin{tabular}{|l|c|c|c|}
\hline \multicolumn{1}{|c|}{ Escalas } & Framingham & REGICOR & ACC/AHA \\
\hline Framingham & $1,00(1,00-1,00)$ & $-0,0811((-0,1664)-(0,0041))$ & $-0,1072((-0,1863)-(0,0282))$ \\
\hline REGICOR & & $1,00(1,00-1,00)$ & $-0,0186((-0,0545)-(0,0174))$ \\
\hline ACC/AHA & & & $1,00(1,00-1,00)$ \\
\hline
\end{tabular}

Fuente: elaboración propia.

$\mathrm{k}=0,4356$ (IC95\%: 0,2675-0,6036); al comparar Framingham con ACC/AHA se obtuvo un $\mathrm{k}=0,3069$ (IC95\%: 0,1831-0,4303), y al comparar REGICOR con ACC/AHA se obtuvo un $\mathrm{k}=0,4791$ (IC95\%: 0,3312$0,6270)$, siendo todas las concordancias de fuerza moderada (Tabla 5). Finalmente, al evaluar la concordancia de riesgo intermedio/moderado entre las escalas Framingham y REGICOR, se obtuvo un $\mathrm{k}=0,3423$ (IC95\%: 0,1753-0,5093), evidenciando también una fuerza de concordancia moderada (Tabla 6).

\section{Discusión}

La patología cardiovascular es la primera causa de muerte en el mundo: según la OMS, citada por Roglic², cerca de 18 millones de personas pierden la vida cada año a causa de esta entidad, lo cual ha motivado a ampliar y fortalecer el conocimiento acerca de los factores de riesgo para presentar alguna enfermedad de este grupo e implementar estrategias como la estratificación de riesgo para impactar de manera temprana en los pacientes y así obtener mejores desenlaces clínicos.

En 2016 se publicó un estudio que analizó diferentes factores de riesgo para la carga mundial de enfermedades, entre ellas la diabetes, y evidenció el alto impacto de estas variables en distintas patologías, encontrando que algunos de los factores modificables como consumo de tabaco, dieta rica en sodio, ingesta excesiva de alcohol y ejercicio físico limitado estuvieron presentes en el $90 \%$ de los pacientes con patología cardiovascular ${ }^{15}$.

Las investigaciones para encontrar la relación entre las escalas de valoración de riesgo cardiovascular se han realizado alrededor del mundo, un ejemplo de estos estudios es el de Gil-Guillen et al. ${ }^{16}$ donde se encontró una concordancia moderada para riesgo alto, lo cual difiere de los hallazgos encontrados en la presente investigación posiblemente debido a la población escogida en el estudio español, que incluyó pacientes

Tabla 5. Concordancia de riesgo bajo en las tres escalas.

\begin{tabular}{|l|c|c|c|}
\hline \multicolumn{1}{|c|}{ Escalas } & Framingham & REGICOR & ACC/AHA \\
\hline Framingham & $1,00(1,00-1,00)$ & $0,4356(0,2675-0,6036)$ & $0,3069(0,1831-0,4303)$ \\
\hline REGICOR & & $1,00(1,00-1,00)$ & $0,4791(0,3312-0,6270)$ \\
\hline ACC/AHA & & & $1,00(1,00-1,00)$ \\
\hline
\end{tabular}

Fuente: elaboración propia.

Tabla 6. Concordancia de riesgo intermedio/moderado en de las escalas Framingham y REGICOR.

\begin{tabular}{|l|c|c|}
\hline \multicolumn{1}{|c|}{ Escalas } & Framingham & REGICOR \\
\hline Framingham & $1,00(1,00-1,00)$ & $0,3423(0,1753-0,5093)$ \\
\hline REGICOR & & $1,00(1,00-1,00)$ \\
\hline
\end{tabular}

Fuente: elaboración propia.

34 Concordancia de tres escalas de riesgo cardiovascular en mujeres con diabetes mellitus tipo 2 e2500-5006 Revista Colombiana de Nefrología 
sin enfermedad cardiovascular establecida; no obstante, allí se describió una mediana de IMC en 25,9, similar a lo aquí reportado.

Posteriormente, Muñoz et al. ${ }^{17}$ estimaron la concordancia entre los modelos de SCORE, Framingham y AHA/CC en una población colombiana y encontraron niveles de colesterol séricos $>200 \mathrm{mg} / \mathrm{dL}$ en el $78 \%$ de las mujeres estudiadas, algo ligeramente distinto a lo encontrado en la población aquí analizada, donde la mediana fue inferior a este valor; los resultados también contrastan respecto a los triglicéridos, pues en dicho estudio los niveles reportados fueron $>150 \mathrm{mg} / \mathrm{dL}$ en el $39 \%$ de la población. Se debe tener en cuenta que solo el $6 \%$ de las mujeres incluidas en el estudio de Muñoz et al. ${ }^{17}$ tenían diagnóstico de diabetes mellitus sin hacer discrepancia en el tipo. De igual forma, es imprescindible hacer énfasis en el hallazgo de pacientes fumadores de dicho estudio ya que es similar al de la presente investigación; esto cobra relevancia cuando se conoce la alta asociación del tabaquismo con la patología cardiovascular y los 7,2 millones de vidas que cobra cada año.

La mediana de edad encontrada en la población del presente estudio se encuentra en el mismo rango que la reportada por Muñoz et al. ${ }^{17} \mathrm{y}$ Strom-Williams et al. ${ }^{18}$.

La hemoglobina glicosilada es una de las variables paraclínicas más importantes en la evaluación de los pacientes con DM2. En Croacia, Sekerija et al. ${ }^{19}$ enrolaron 8.775 pacientes con DM2, de los cuales 4.197 eran mujeres, e identificaron un mediana de hemoglobina glicosilada en el 7,05\% de este grupo, hallazgo que coincide con lo reportado en el presente estudio y describe un control metabólico aceptable en población femenina con DM2. Cabe resaltar que en el estudio realizado en Croacia se encontró un mejor control metabólico en pacientes masculinos ${ }^{19}$.

Es importante resaltar que en los estudios de concordancia de escalas de riesgo cardiovascular realizados en Colombia no se ha descrito el tiempo de ejercicio diario que realiza cada paciente, a pesar de ser este uno de los factores de riesgo más relevantes en esta entidad; por otra parte, no hay ninguna descripción del estrato socioeconómico de los pacientes, con lo que se podría definir la relación de estas patologías con los rangos salariales en Colombia, siendo esto un dato útil en la evaluación de cada paciente.

En diversos estudios ${ }^{20,21}$ realizados en Latinoamérica se ha encontrado pobre concordancia entre las escalas utilizadas para evaluar riesgo cardiovascular, pero específicamente en Colombia no hay investigaciones donde se valore la concordancia de estas ayudas de estratificación de riesgo directamente en mujeres con DM2, lo cual se ve reflejado directamente en la toma de decisiones sobre esta población.

\section{Conclusiones}

En el presente estudio se observó una baja concordancia para riesgo cardiovascular alto al comparar las escalas Framingham, SCORE/REGICOR y ACC/ AHA en mujeres colombianas con DM2; por el contrario, se evidenció una concordancia moderada cuando se evaluaron estas mismas escalas para riesgo cardiovascular bajo. La edad y el colesterol total fueron los valores que más afectaron la variación entre la escala de riesgo en comparación.

\section{Conflicto de intereses}

Ninguno declarado por los autores.

\section{Financiación}

Este estudio recibió apoyo financiero por la Coordenação de Aperfeiçoamento de Pessoal de Nível Superior - Brasil (CAPES) - Código de Finanzas 001.

\section{Agradecimientos}

Ninguno declarado por los autores.

\section{Contribución de los autores}

Todos los autores tuvieron igual contribución en la elaboración del documento, por todo los demás estamos de acuerdo con el documento. 
Rev. Colomb. Nefrol. 2020;7(2):30-36, julio-diciembre de 2020 http://www.revistanefrologia.org

http://dx.doi.org/10.22265/acnef.7.2.429

\section{Referencias}

1. Flor LS, Campos MR. The prevalence of diabetes mellitus and its associated factors in the Brazilian adult population: evidence from a population-based survey. Rev Bras Epidemiol. 2017;20(1):16-29. http://dx.doi.org/10.1590/1980-5497201700010002.

2. Roglic G. WHO Global report on diabetes: A summary. Int J Non-Commun Dis. 2016;1(1):3-8.

3. Vargas-Uricoechea H, Casas-Figueroa LÁ. Epidemiología de la diabetes mellitus en Sudamérica: la experiencia de Colombia. Clin Investig Arterioscler. 2016;28(5):245-56. https://doi.org/10.1016/j.arteri.2015.12.002.

4. Selvin E, Lazo M, Chen Y, Shen L, Rubin J, McEvoy JW, et al. Diabetes mellitus, prediabetes, and incidence of subclinical myocardial damage. Circulation. 2014;130(16):1374-82. http://dx.doi.org/10.1161/CIRCULATIONAHA.114.010815.

5. Mason JC, Libby P. Cardiovascular disease in patients with chronic inflammation: mechanisms underlying premature cardiovascular events in rheumatologic conditions. Eur Heart J. 2015;36(8):482-9c. http://dx.doi.org/10.1093/eurheartj/ehu403.

6. Minoo F, Mahdavi-Mazdeh M, Abbasi MR, Sohrabi S. Impact of the severity of obesity on microalbuminuria in obese normotensive nondiabetic individuals. J Ren Inj Prev. 2015;4(2):34-8. http://dx.doi.org/10.12861/jrip.2015.08.

7. Wu Y, Ding Y, Tanaka Y, Zhang W. Risk factors contributing to type 2 diabetes and recent advances in the treatment and prevention. Int J Med Sci. 2014;11(11):1185-200. http://dx.doi.org/10.7150/ijms.10001.

8. D’Agostino RB, Pencina MJ, Massaro JM, Coady S. Cardiovascular Disease Risk Assessment: Insights from Framingham. Glob Heart. 2013;8(1):11-23. http://dx.doi.org/10.1016/j.gheart.2013.01.001.

9. Gabriel R, Brotons C, Tormo MJ, Segura A, Rigo F, Elosua R, et al. The ERICE-score: the new native cardiovascular score for the lowrisk and aged Mediterranean population of Spain. Rev Esp Cardiol (Engl Ed). 2015;68(3):205-15. http://dx.doi.org/10.1016/j.rec.2014.03.019.

10. VandenBos GR, Winkler JM. An Analysis of the Status of Journals and Research in Psychology from Latin America. Psicol Reflex E Crítica. 2015;28(Suppl 1):82-93. https://doi.org/10.1590/1678-7153.20152840012.

11. Camacho PA, Otero J, Pérez M, Arcos E, García H, Narvaez C, et al. The spectrum of the dyslipidemia in Colombia: The PURE study. Int J Cardiol. 2019;284:111-7. https://doi.org/10.1016/j.ijcard.2018.10.090.

12. López-Jaramillo P, Calderón C, Castillo J, Escobar ID, Melgarejo E, Parra GA. Prediabetes in Colombia: Expert Consensus. Colomb Médica. 2017;48(4):191-203. http://dx.doi.org/10.25100/cm.v43i4.3662.

13. Navarrete S, Huertas D, Rozo JE, Ospina JE. Prevalencia de factores de riesgo para enfermedad cardiovascular en una muestra de pacientes con hipertensión arterial esencial: estudio descriptivo. Rev Colomb Cardiol. 2009;16(2):64-70.

14. Machado-Alba JE, Machado-Duque ME. Prevalencia de factores de riesgo cardiovascular en pacientes con dislipidemia afiliados al sistema de salud en Colombia. Rev Peru Med Exp Salud Publica. 2013 [cited abril 11 2020];30(2):205-11. Disponible en: http://www.scielo.org.pe/scielo.php?script=sci_abstract\&pid=S1726-46342013000200007\&lng=es\&nrm=iso\&tlng=es.

15. GBD 2015 Risk Factors Collaborators. Global, regional, and national comparative risk assessment of 79 behavioural, environmental and occupational, and metabolic risks or clusters of risks, 1990-2015: a systematic analysis for the Global Burden of Disease Study 2015. Lancet. 2016;388(10053):1659-724.https://dx.doi.org/10.1016/S0140-6736(16)31679-8.

16. Gil-Guillén V, Orozco-Beltrán D, Maiques-Galán A, Aznar-Vicente J, Navarro J, Cea-Calvo L, et al. Concordancia de las escalas REGICOR y SCORE para la identificación del riesgo cardiovascular alto en la población española. Rev Esp Cardiol. 2007;60(10):104250. http://dx.doi.org/10.1157/13111236.

17. Muñoz OM, Ruiz-Morales ÁJ, Mariño-Correa A, Bustos MM. Concordancia entre los modelos de SCORE y Framingham y las ecuaciones AHA/ACC como evaluadores de riesgo cardiovascular. Rev Colomb Cardiol. 2017;24(2):110-6.

https://doi.org/10.1016/j.rccar.2016.06.013.

18. Strom-Williams JL, Lynch CP, Winchester R, Thomas L, Keith B, Egede LE. Gender differences in composite control of cardiovascular risk factors among patients with type 2 diabetes. Diabetes Technol Ther. 2014;16(7):421-7. https://doi.org/10.1089/dia.2013.0329.

19. Sekerija M, Poljicanin T, Erjavec K, Liberati-Cizmek AM, Prašek M, Metelko Z. Gender differences in the control of cardiovascular risk factors in patients with type 2 diabetes -a cross-sectional study. Intern Med Tokyo Jpn. 2012;51(2):161-6. https://doi.org/10.2169/internalmedicine.51.6094.

20. Lister-Del Pino P, León-Amenero G, Leiva-Montejo A, Segura ER. Concordancia entre las escalas de riesgo cardiovascular Procam y Framingham en varones que reciben tratamiento antirretroviral en un Hospital Nacional de Lima, Perú 2013. Rev Peru Med Exp Salud Publica. 2015;32(4):731-8.

21. García-Valenzuela SM. Concordancia en la evaluación del riesgo cardiovascular entre las escalas Score, Framingham y AHA/ACC en pacientes con dislipidemia en un hospital de altura [tesis]. Trujillo: Universidad Privada Antenor Orrego; 2019 [citado noviembre 20 2020]. Disponible en: http://repositorio.upao.edu.pe/handle/upaorep/4533.

36 Concordancia de tres escalas de riesgo cardiovascular en mujeres con diabetes mellitus tipo 2 e2500-5006 Revista Colombiana de Nefrología 